Check for updates

Cite this: RSC Adv., 2018, 8, 22536

Received 14th March 2018

Accepted 6th June 2018

DOI: $10.1039 / c 8 r a 02236 d$

rsc.li/rsc-advances

\section{Acid green crystal-based in situ synthesis of polyaniline hollow nanotubes for the adsorption of anionic and cationic dyes $\uparrow$}

\author{
Wael A. Amer, (D) ${ }^{a}$ Mohamed M. Omran, ${ }^{a}$ Ahmed F. Rehab ${ }^{a}$ \\ and Mohamad M. Ayad (D) *ab
}

In this article, acid green (AG) dye played a dual pivotal role. The first role is as a structure-guiding agent and a soft template for the acid-free synthesis of polyaniline hollow nanotubes (PANI-HNTs) while the second role is as a target dye to be removed as a model of anionic dyes, alongside methylene blue (MB) as a model of cationic dyes. After characterization using X-ray diffraction, Fourier transform infrared spectroscopy, transmission electron microscopy (TEM), nitrogen adsorption-desorption isotherms (BET), and UV-visible absorption spectroscopy techniques, the prepared PANI-HNTs were exploited for the adsorption of basic MB dye and acidic AG dye from aqueous media. To optimize the adsorption process, the effects of different variables, such as adsorbent dose, contact time and $\mathrm{pH}$, were studied. Due to the structural differences between the MB and AG dyes, the obtained data revealed that the best $\mathrm{pH}$ for the medium for optimal adsorption was 9.0 and 3.0, respectively. The rapid sorption dynamics were found to proceed in a second-order kinetic model and the equilibrium data for the adsorption of MB and AG dyes were fitted well to the Langmuir isotherm. The maximum monolayer capacity, $q_{\text {max }}$, for MB and AG was calculated from the Langmuir model and equalled 69.4 and $57.8 \mathrm{mg} \mathrm{g}^{-1}$, respectively. A thermodynamic study revealed that the adsorption of MB by PANI-HNTs was a feasible, spontaneous, and exothermic process. Investigation of the substrate regeneration revealed that PANI-HNTs can be reused for dye adsorption several times. Therefore, the synthesized PANI-NTs are highly efficient for the dual removal of basic and acidic dyes. TEM images showed that PANI-HNTs were formed with an external and internal diameter of 50-60 $\mathrm{nm}$ and 5-10 $\mathrm{nm}$, respectively.

\section{Introduction}

Industrial wastewater may contain some toxic dyes, such as acidic and basic dyes. ${ }^{1}$ The release of toxic dyes by the textile, dyestuffs, leather, paper, foodstuffs, cosmetics, rubber, plastics and paint industries into the environment poses a risk to human health., ${ }^{2,3}$ Due to their chemical structure, dyes are resistant to many chemical reagents, oxidizing agents, and heat, and are nonbiodegradable. ${ }^{4}$ Hence, decolorizing the effluents once they have been released into the aquatic environment is a big challenge. The most important methods available for the removal of dyes from wastewater are chemical coagulation, Fenton oxidation, ozonation, membrane processes and adsorption. ${ }^{\mathbf{1 , 2 , 5}}$

Adsorption is an effective technique that is widely used for the treatment of dyes in wastewater ${ }^{\mathbf{1 , 4}, \mathbf{6}}$ due to its ease of use and

${ }^{a}$ Chemistry Department, Faculty of Science, Tanta University, Tanta 31527, Egypt. E-mail: mohamad.ayad@ejust.edu.eg; Fax: +20 3459 9520; Tel: +20 34599520

${ }^{b}$ Institute of Basic and Applied Sciences, Egypt-Japan University of Science and Technology, New Borg El-Arab City, Alexandria 21934, Egypt

$\dagger$ Electronic supplementary information (ESI) available. See DOI: $10.1039 / \mathrm{c} 8 \mathrm{ra02236d}$ inexpensive nature. ${ }^{2}$ Due to its high adsorption efficiency, activated carbon has been extensively used as an adsorbent, but a problem lies in its high usage costs, ${ }^{2,3,6}$ which led many researchers to look for inexpensive and locally available alternative adsorbents to render the adsorption process economically feasible. ${ }^{5}$

Polyaniline (PANI) has attracted much attention due to its unique properties and advantages, such as its easy synthesis, good environmental stability and low cost. ${ }^{3,7-11}$ PANI can exist in three different isolable oxidation states at the molecular level; the leucoemeraldine, emeraldine and pernigraniline states. ${ }^{\mathbf{1 2}}$ Other oxidation states are the result of physical mixtures of these oxidation states. The emeraldine base is the intermediate oxidation state of PANI and is composed of equal amounts of alternating reduced base and oxidized base units. ${ }^{12,13}$

Nanostructured PANI has received a great deal of attention due to its high surface area and fantastic properties, which differ from those of the coarse structures. While conventional PANI powder shows a limited adsorption of dyes, ${ }^{14-16}$ nanostructured PANI has an improved adsorption capacity for dyes. ${ }^{5}$

As a form of PANI nanostructure, PANI nanotubes have been used effectively for the removal of $\mathrm{MB}$ as a cationic dye and acid 
green (AG) as an anionic dye from aqueous solutions. ${ }^{8,17}$ PANINTs base/silica composites and nanostructured crosslinked PANI were found to have little adsorption capacity for dyes. ${ }^{18,19}$ Furthermore, PANI nanostructures with different morphologies have only been exploited for the adsorption of anionic acid blue 25 dye $\left(q_{\max }=70 \mathrm{mg} \mathrm{g}^{-1}\right){ }^{20}$

Many methods can be utilized for the synthesis of PANI nanostructures, such as electrospinning, interfacial polymerization, electrochemical polymerization and template-synthesis (with either a soft or hard template). ${ }^{21-26}$

The diameter of the synthesized nanostructures, using the hard-template method, depends on the pore diameter of the hard template. ${ }^{27}$ Although many materials have been exploited as hard templates, such as zeolites with channels, inorganic aluminum oxide, and porous polymer membranes, ${ }^{26}$ posttreatment is necessary for the removal of the template, which in turn renders the preparation process more complex and may damage the nanostructures. ${ }^{9,27}$

Additionally, PANI-NTs have been synthesized via the softtemplate method using structure-guiding molecules, such as surfactant micelles ${ }^{28}$ organic acids ${ }^{29}$ and reverse micro-emulsions. ${ }^{30}$ These materials can form, on their own or with aniline, different forms of structural aggregates such as cylindrical micelles. ${ }^{9}$ Afterwards, the polymerization process is initiated via the addition of a chemical oxidant such as ammonium peroxydisulfate (APS) or ferric chloride to form PANI-NTs. The major advantages of the soft template method lie in its simplicity, large yield, low cost, and the absence of a post-treatment step as well. $^{26,31}$

A very limited number of anionic dyes have been exploited in the synthesis of PANI nanostructures, such as acid red 8 crystals (in the presence of hydrochloric acid and $\mathrm{APS}^{32}$ ) and methyl orange (MO) (used in the presence of hydrochloric acid and APS as an oxidant to synthesize PANI microtubes with an average diameter of $350 \mathrm{~nm}$ ). ${ }^{26}$ Furthermore, PANI microtubes doped with MO have been synthesized with an inner diameter and outer diameter of about $800 \mathrm{~nm}$ and $1.5 \mu \mathrm{m}$, respectively. ${ }^{33}$

Studies on the AG crystal-based in situ synthesis of PANI hollow nanotubes (PANI-HNTs) are lacking in the literature and hence, this was the first aim of the current work. Moreover, the synthesized nanostructures were exploited for the dual adsorption of cationic and anionic dyes.

Here, PANI-HNTs were synthesized via the in situ polymerization of aniline using APS as an oxidant in aqueous solution in the presence of AG crystals as a soft-template, without using any acid or surfactants. In order to ensure the removal of the AG template and the oligomers, Soxhlet extraction was performed.

After characterization using Fourier transform infrared (FTIR) spectroscopy, X-ray diffraction (XRD), UV-vis absorption spectroscopy and transmission electron microscopy (TEM), the role of PANI-HNTs as an adsorbent (of cationic and anionic dyes) was examined. The effect of $\mathrm{pH}$, contact time, adsorbent dosage, and initial dye concentration on the adsorption of dyes was investigated. Moreover, the adsorption properties of the PANI-NTs were examined from a kinetic and isotherm point of view.

\section{Experimental}

\subsection{Materials}

Aniline (ADWIC, Egypt) was distilled using zinc dust. ${ }^{34}$ The other chemicals, such as glacial acetic acid, boric acid and sodium hydroxide, were bought from ADWIC, Egypt, and were used as received. Ammonium peroxydisulfate (APS), phosphoric acid, MB and AG from Sigma-Aldrich were used without further purification. All chemicals were of analytical grade. Solutions were prepared using freshly distilled water.

\subsection{Synthesis of PANI-HNTs}

PANI-HNTs were synthesized according to previous reports. ${ }^{35,36}$ $0.3125 \mathrm{~g}(0.5 \mathrm{mmol})$ of AG was dissolved in distilled water (60 $\mathrm{mL})$ at $5{ }^{\circ} \mathrm{C}$ and then $0.93 \mathrm{~g}(10 \mathrm{mmol})$ of aniline was added to the AG solution. A precooled solution of $2.8525 \mathrm{~g}(12.5 \mathrm{mmol})$ APS in distilled water $(40 \mathrm{~mL})$ was added drop wise to the aniline-AG solution. The resulting mixture was kept under stirring for $18 \mathrm{~h}$. The resulting precipitate was separated and purified via Soxhlet extraction with acetone to remove the AG and oligomers, rinsed with ethanol and dried at $50{ }^{\circ} \mathrm{C}$. A series of PANI-AG samples was prepared by varying the molar ratio of aniline to AG from 10 to 20 so as to investigate the optimum synthesis conditions.

\subsection{Adsorption of dyes}

In batch adsorption experiments, $100 \mathrm{~mL}$ aqueous $\mathrm{MB}$ and $\mathrm{AG}$ solutions were mixed with PANI-HNTs and were magnetically stirred at $300 \mathrm{rpm}$ in $\mathrm{pH} \mathrm{3,7}$ and 9 buffered solutions at room temperature. The separation of the adsorbent was done at predetermined time intervals via centrifugation at $4000 \mathrm{rpm}$ for $10 \mathrm{~min}$ and then the absorbance was measured at $664 \mathrm{~nm}$ and $642 \mathrm{~nm}$ for the MB and AG dyes, respectively.

The buffer solutions were prepared in the range 1.81-11.98 by adding $0.2 \mathrm{~N}$ sodium hydroxide solution to $100 \mathrm{~mL}$ of a phosphoric acid, acetic acid and boric acid solution (each 0.04 $\mathrm{M}$ ) and the final $\mathrm{pH}$ was adjusted with a $\mathrm{pH}$ meter (JENWAY $3510)^{37}$

The dye removal percentage was determined as follows: ${ }^{1,38}$

$$
\text { Removal percentage }=100\left(C_{\mathrm{o}}-C_{t}\right) / C_{\mathrm{o}}
$$

The dye uptake was determined as follows: ${ }^{8}$

$$
q_{t}=\left(C_{\mathrm{o}}-C_{t}\right) V / W
$$

where, $q_{t}\left(\mathrm{mg} \mathrm{g}^{-1}\right)$ is the amount of adsorbed dye per unit mass of the adsorbent at time $t(\mathrm{~min}), C_{\mathrm{o}}\left(\mathrm{mg} \mathrm{L}^{-1}\right)$ and $C_{t}\left(\mathrm{mg} \mathrm{L}^{-1}\right)$ are the dye concentrations at time 0 and $t, V(\mathrm{~L})$ is the solution volume, and $W(\mathrm{~g})$ is the adsorbent mass.

\subsection{Instruments}

Transmission electron microscope (TEM) images were obtained using a JEM-2100F at $200 \mathrm{kV}$. X-ray diffraction (XRD) patterns were obtained using a GNR APD-2000 PRO diffractometer with 
$\mathrm{Cu} \mathrm{K} \alpha$ radiation $(40 \mathrm{kV}, 30 \mathrm{~mA})$ in a step scan mode. FT-IR spectra were recorded using a Thermo Scientific NICOLET IS 50 FT-IR spectrophotometer with a frequency range of 4000$400 \mathrm{~cm}^{-1}$. The Brunauer-Emmett-Teller (BET) surface area, Barrett-Joyner-Halenda (BJH) pore size and pore size distributions of the materials were measured through nitrogen adsorption using a surface area and porosity analyzer (Micromeritics, ASAP 2020). The optical absorption spectra were recorded using a UV spectrometer, UVD-2960 (Labomed Inc.).

\section{Results and discussion}

\subsection{Synthesis of PANI-HNTs}

In a saturated anionic dye (AG) solution, the solubility of AG decreases leading to crystallization into crystals at the microscale, which were suspended in the solution. ${ }^{36}$ In addition, aniline (An) monomers have low solubility and will be adsorbed onto the surface of the anionic AG crystals. These An-AG dye crystals can offer substrates for other An monomers to adsorb and grow on. An monomers are ready to polymerize on the surface of the An-AG dye crystals upon the addition of an APS solution. The polymerization reaction ends with the formation of a PANI shell with AG as the core template. The resulting product is purified from the AG crystal cores and the residual reactants and oligomers via Soxhlet extraction with acetone, as shown in Scheme 1.

After the formation and drying of PANI-HNTs, the FTIR spectrum was measured. The spectrum is shown in Fig. 1, with the main characteristic IR peaks at 3240, 2940, 1590, 1490, 1300, 1140, 1040, 815 and $695 \mathrm{~cm}^{-1}$. The obtained characteristic absorption bands are tabulated in Table 1 .

TEM images were recorded to gain more insight into the structure of the formed PANI-HNTs and their morphology. Fig. 2 shows the TEM images of the PANI-HNTs formed using different molar ratios of An to AG to investigate the effect of the molar ratio on the morphology of the resulting PANIHNTs. When the molar ratio of An : AG was 10 (Fig. 2A), it was found that the morphology of PANI was interconnected nanorods. The nanotubular morphology of PANI started to appear on a small scale (Fig. 2B) when the molar ratio was 15 but when the molar ratio was 20 (Fig. 2C and D), PANI-HNTs were obtained with outer and inner diameters of about 60 and $5 \mathrm{~nm}$, respectively. These findings confirm that AG crystals acted as the core and An monomers were regularly

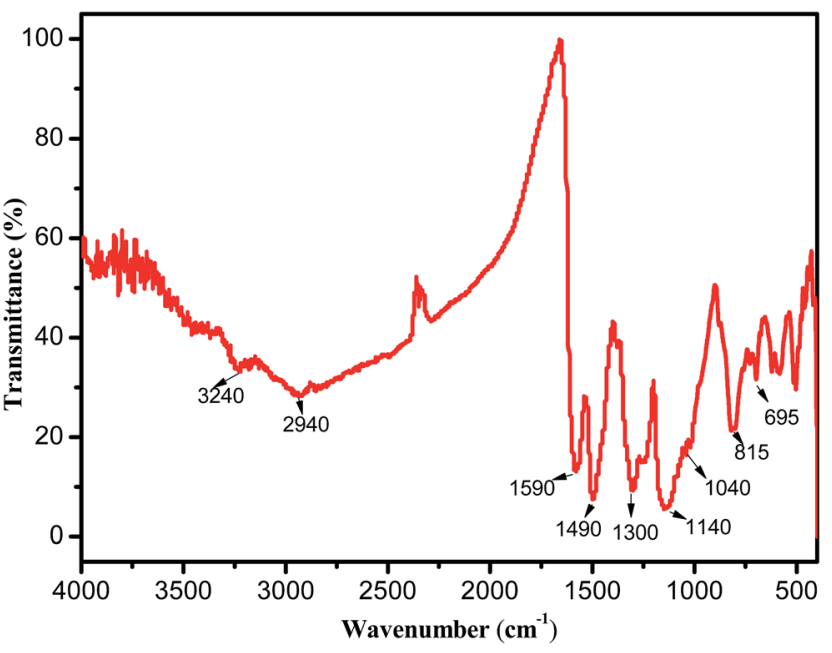

Fig. 1 FTIR spectrum of PANI-HNTs salt.

polymerized onto the AG surface, which led to the construction of the core-shell structure that eventually led to the formation of hollow nanotubes after the removal of the core AG via Soxhlet extraction.

Fig. 3 shows the XRD patterns of the conventional PANI (PANI con) and PANI-AG prepared with different molar ratios of An : AG. It is observed that PANI undergoes interfacial interaction with AG and exhibits semi-crystalline behavior. PANI con and PANI-AG show peaks indicating that AG crystallites have been uniformly mixed within the polymer chain during the polymerization.

Nitrogen adsorption-desorption isotherms of PANI-HNTs were measured and are shown in Fig. 4, giving characteristic information about the specific surface area $\left(S_{\mathrm{BET}}\right)$, the pore size $(D)$ and the total pore volume $\left(V_{\mathrm{t}}\right)$ of the synthesized PANIHNTs. According to the IUPAC classification, PANI-HNTs exhibited type-IV isotherms. ${ }^{42}$ The collected data are summarized in Table 2. The specific surface areas of PANIHNTs (39.18 $\mathrm{m}^{2} \mathrm{~g}^{-1}$ ) calculated using the BET equation were found to be larger than that of conventional PANI. ${ }^{19}$ Furthermore, the pore volume $\left(V_{\mathrm{t}}\right)$ calculated using the BJH method is about $0.072 \mathrm{~cm}^{3} \mathrm{~g}^{-1}$ and the mean pore diameter was found to be $7.4 \mathrm{~nm}$. The larger surface area found is expected to impart the synthesized PANI-HNTs with good adsorption properties.

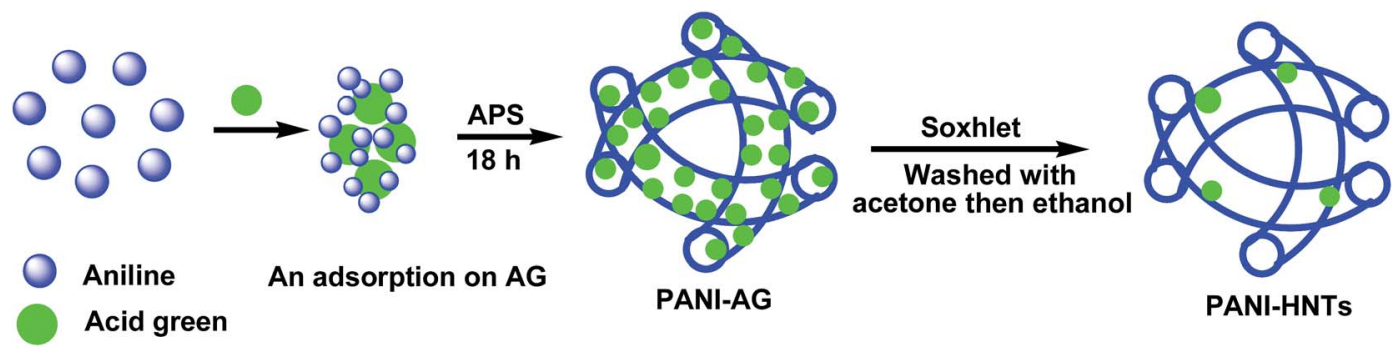

Scheme 1 Schematic diagram of PANI-HNTs formation. 
Table 1 FTIR data for PANI-HNTs

\begin{tabular}{ll}
\hline Wavenumber, $\mathrm{cm}^{-1}$ & Peak assignment \\
\hline 3240 & Hydrogen-bonded N-H stretching vibration \\
1590 & PANI quinoid (Q) ring stretching \\
1490 & Benzenoid (B) ring stretching \\
1300 & C-N stretching of secondary aromatic amine \\
1140 & Aromatic C-H in-plane bending \\
1040 and 695 & S=O and S-O stretching vibration modes \\
& of the sulfonate groups attached to the \\
& aromatic rings
\end{tabular}

\subsection{Adsorption of MB and AG onto PANI-NTs}

3.2.1. Effect of $\mathbf{p H}$ and adsorption mechanism. The oxidation states and hence the properties of PANI depend mainly on the $\mathrm{pH}$ of the medium. To find the optimum $\mathrm{pH}$ conditions for the dual adsorption of dyes ( $\mathrm{MB}$ and $\mathrm{AG}$ ), the adsorption of dyes was carried out at $\mathrm{pH} 3$, 5, 7, 9 and 11. Fig. 5A shows the percentage removal of MB dye using PANI-HNTs at the estimated $\mathrm{pH}$ values. $20 \%$ and $47 \%$ of $\mathrm{MB}$ dye was removed after 7 hours at pH 3 and 5, respectively, while $82 \%, 88 \%$ and $91 \%$ of MB dye was removed after 10 hours when the $\mathrm{pH}$ was raised to 7, 9 and 11, respectively. For the removal of the anionic AG dye, as shown in Fig. 5B, the percentage removal of AG dye was found to be $0 \%$ at $\mathrm{pH} 11$ and 9 , and $5 \%$ at $\mathrm{pH} 7$, while it was about $35 \%$ and $52 \%$ at $\mathrm{pH}=5$ and 3 after 6 hours, respectively. It is clearly shown that the PANI-HNTs have a high adsorption capacity for $\mathrm{MB}$ at $\mathrm{pH} 11\left(69.4 \mathrm{mg} \mathrm{g}^{-1}\right)$ and an adsorption capacity of $57.8 \mathrm{mg} \mathrm{g}^{-1}$ for AG at $\mathrm{pH}$ 3. Therefore, the optimum $\mathrm{pH}$ values for the adsorption of $\mathrm{MB}$ as a cationic dye and $\mathrm{AG}$ as

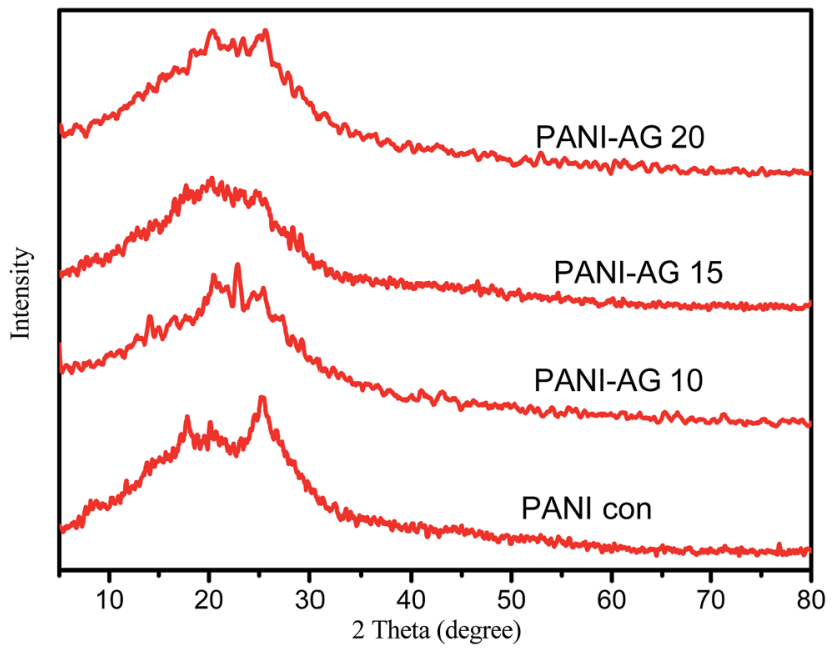

Fig. 3 XRD patterns of PANI con and PANI-AG with different molar ratios, $A n: A G=10, A n: A G=15$ and $A n: A G=20$.

an anionic dye using PANI-NTs are $\mathrm{pH} 11$ and 3, respectively. All the subsequent studies in this work were carried out at $\mathrm{pH}$ values of 3 and 9 .

The AG adsorption on PANI-HNTs at $\mathrm{pH} 3$ can be attributed to the electrostatic interaction between the positively charged $-\mathrm{NH}_{2}{ }^{+}$groups of the adsorbent PANI-HNTs surface (due to the acidic $\mathrm{pH} 3$ ) and the anionic AG dye. At low pH values, very low electrostatic attraction exists between the adsorbent PANI-HNTs surface and MB because of the presence of positively charged sites on both surfaces and the lack of free unpaired electrons on the adsorbent surface and hence acidic low $\mathrm{pH}$ values do not
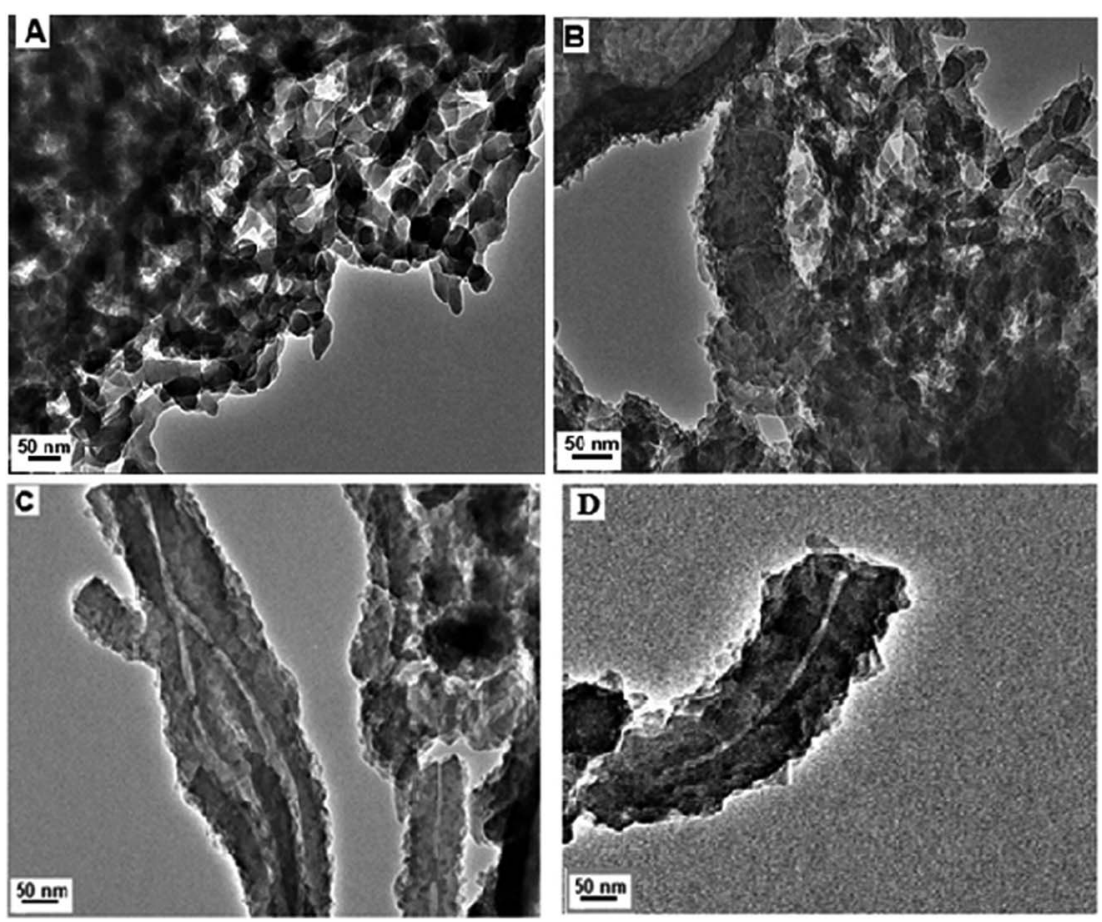

Fig. 2 TEM images of the resulting PANI at different molar ratios, (A) An : AG =10, (B) An : AG =15, (C) and (D) An : AG = 20. 

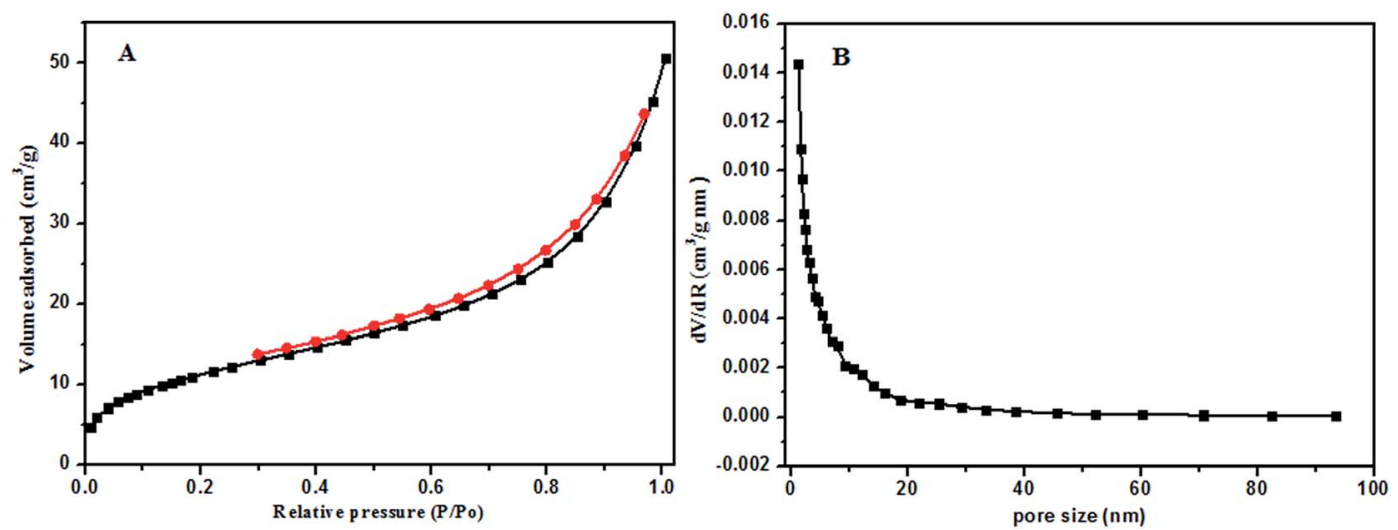

Fig. 4 (A) Nitrogen adsorption-desorption isotherm and (B) pore size distribution curve of PANI-HNTs.

Table 2 Specific surface area and pore volume of the conventional PANI and PANI-HNTs

\begin{tabular}{llll}
\hline Sample & $\begin{array}{l}\text { Specific surface } \\
\text { area }\left(\mathrm{m}^{2} \mathrm{~g}^{-1}\right)\end{array}$ & $\begin{array}{l}\text { Pore volume } \\
\left(\mathrm{cm}^{3} \mathrm{~g}^{-1}\right)\end{array}$ & Reference \\
\hline $\begin{array}{llll}\text { Conventional PANI } \\
\text { PANI-HNTs }\end{array}$ & 20.02 & 0.0071 & 19 \\
& 39.18 & 0.072 & This work
\end{tabular}

favor the adsorption of the cationic MB dye. As the $\mathrm{pH}$ of the system increases, free unpaired electrons become available on the adsorbent surface, which in turn will increase the electrostatic attraction forces between $\mathrm{MB}$ and the adsorbent sites resulting in a high adsorption capacity of $\mathrm{MB}$ at high $\mathrm{pH}$ values. A summary of this adsorption mechanism at pH 3 and 9 is shown in Scheme 2.

3.2.2. Contact time effect. The influence of contact time on the adsorption of the cationic MB dye and the anionic AG dye was examined by measuring the UV-vis absorption spectra for MB and AG solutions (Fig. 6 and $\mathrm{S} 1 \dagger$ ). It was observed that the absorbance of $\mathrm{MB}$ and $\mathrm{AG}$ dye solutions decreases with increasing time, as shown in Fig. $6 \mathrm{~A}$ and $\mathrm{S} 1 \mathrm{~A}, \dagger$ respectively. To show the effect of contact time on the concentration of dyes, the
$\mathrm{MB}$ and AG concentration was plotted against time (Fig. 6B and $\mathrm{S} 1 \mathrm{~B} \dagger)$. The rate of dye adsorption by the PANI-HNTs substrate was observed to be very rapid for an initial period of $20 \mathrm{~min}$ for the MB and AG dye, which indicates the high ability and efficiency of PANI-HNTs for the removal of MB and AG. Thereafter, the dye adsorption processes proceeded at a slower rate and finally reached the equilibrium state within $480 \mathrm{~min}(8 \mathrm{~h})$ for $\mathrm{MB}$ and $240 \mathrm{~min}(4 \mathrm{~h})$ for AG. The slow rate of dye adsorption at long times may result from the partial coverage and saturation of the PANI-HNTs with the dyes.

3.2.3. Effect of different initial dye concentrations. The effect of the initial $\mathrm{MB}$ and $\mathrm{AG}$ dye concentration on their removal efficiency using the PANI-HNTs substrate was investigated at pH 9 and 3 (Fig. 7), respectively. As observed from both figures, the efficiency of dye removal decreases with increasing the initial dye concentration, possibly as a consequence of the surface coverage of PANI-HNTs by the dye molecules.

\subsection{Kinetic study}

In order to study the dye adsorption kinetics, pseudo-firstorder, ${ }^{\mathbf{4 3}}$ pseudo-second-order ${ }^{\mathbf{4 4}}$ and intraparticle diffusion models ${ }^{45}$ were examined.
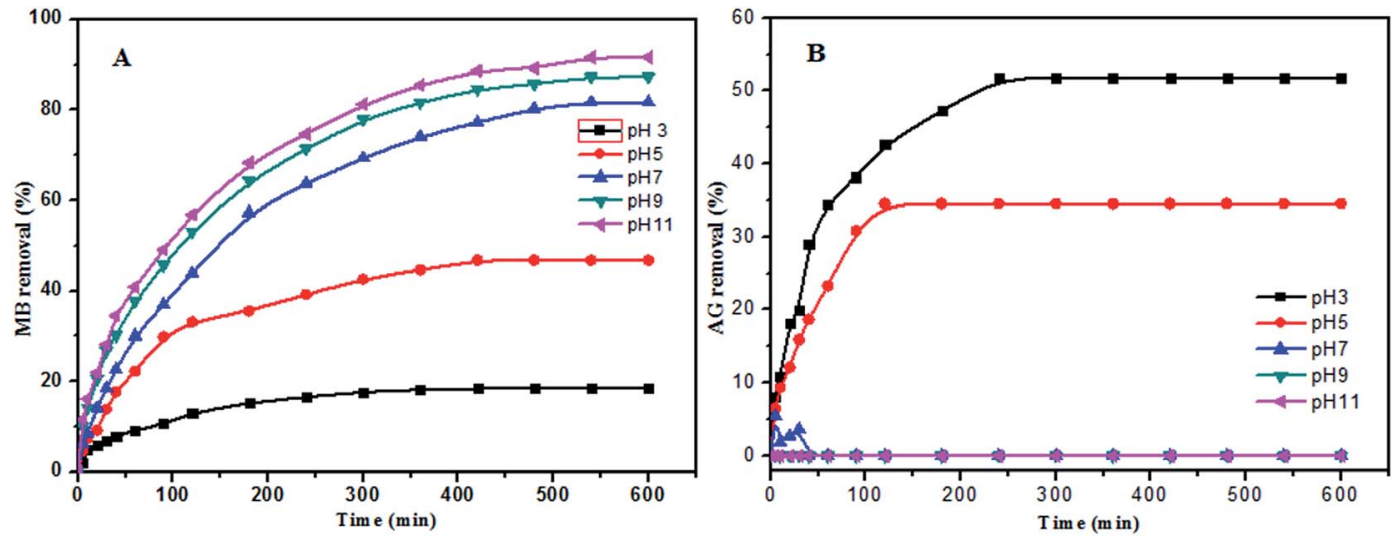

Fig. 5 (A) Effect of $\mathrm{pH}$ on the removal of $\mathrm{MB}$ dye (PANI-HNTs $\left.=10 \mathrm{mg},[\mathrm{MB}]=6.2 \mathrm{mg} \mathrm{L}^{-1}\right)$, (B) effect of $\mathrm{pH}$ on the removal of AG dye (PANI-NTs $=$ $10 \mathrm{mg},[\mathrm{AG}]=6.1 \mathrm{mg} \mathrm{L}^{-1}$ ). 


.

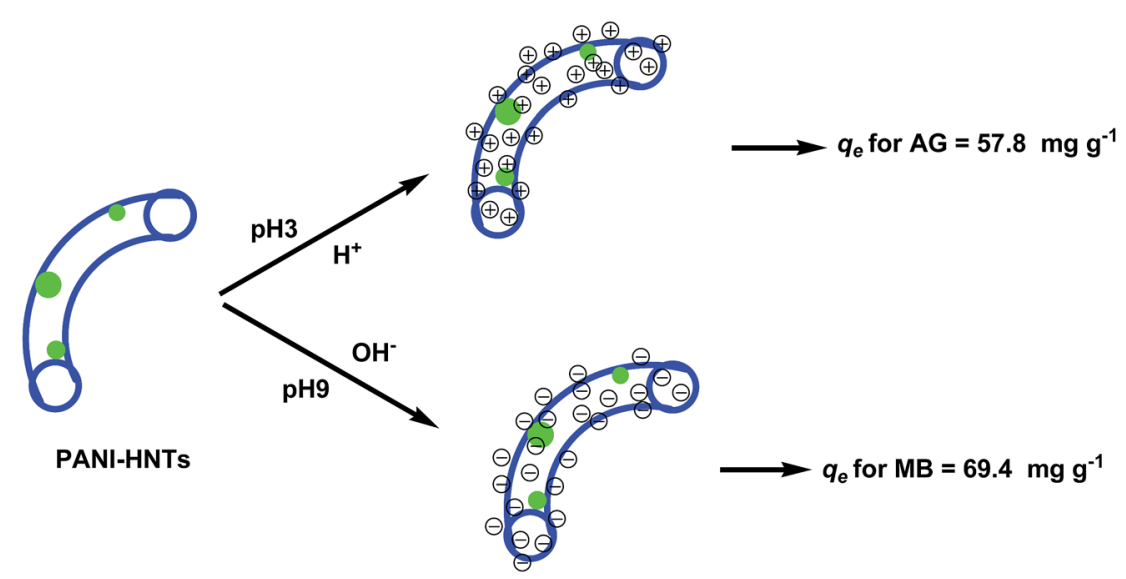

Scheme 2 Schematic diagram of PANI-HNTs at pH 3 and 9 (blue color: PANI-HNTs, green color: AG).

The pseudo-first-order rate kinetics is given by the equation: $\mathbf{1}^{1,38}$

$$
\log \left(q_{\mathrm{e}}-q_{t}\right)=\log q_{\mathrm{e}}-\frac{k_{\mathrm{ad}}}{2.303} t
$$

The pseudo-second-order kinetics is given by the equation: $:^{1,438}$

$$
\frac{t}{q_{t}}=\frac{1}{k_{\mathrm{s}} q_{\mathrm{e}}{ }^{2}}+\frac{t}{q_{\mathrm{e}}}
$$

The intraparticle diffusion model can be expressed as follows: $:^{1,38}$

$$
q_{t}=k_{\mathrm{p}} t^{\frac{1}{2}}+C
$$

where $q_{\mathrm{e}}$ and $q_{t}$ are the amounts of dye adsorbed at equilibrium and time $t$, respectively. $k_{\mathrm{ad}}, k_{\mathrm{s}}$ and $k_{\mathrm{p}}$ are the rate constants of the pseudo-first order, pseudo-second order and intraparticle diffusion models. $C$ is a constant. The validity of the models was verified using the linear equation analysis of $\log \left(q_{\mathrm{e}}-q_{t}\right)$ vs. $t,(t)$ $\left.q_{t}\right)$ vs. $t$ and $q_{t} v s . t^{1 / 2}$, respectively. The kinetic parameters of the pseudo-first order, pseudo-second order, and intraparticle

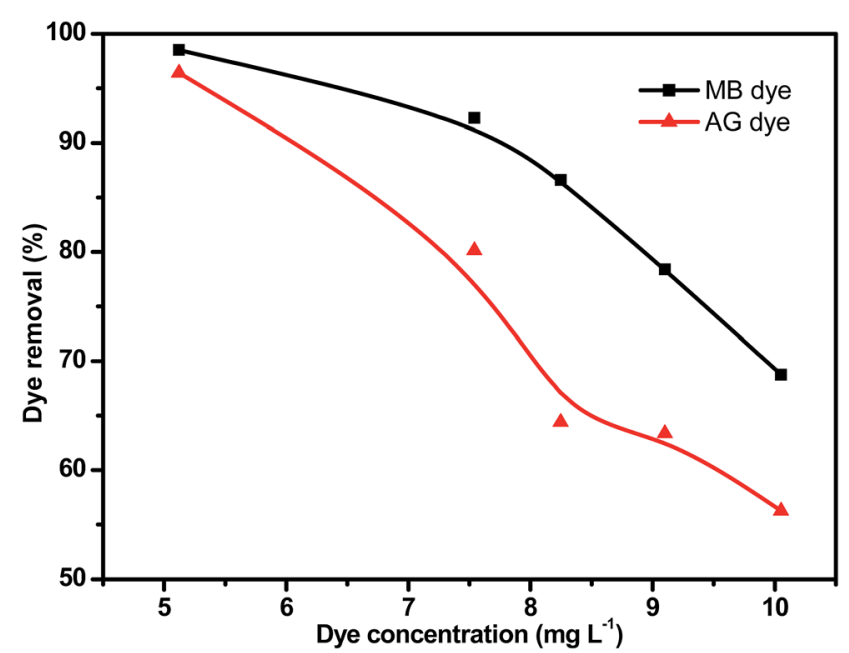

Fig. 7 Effect of initial concentration of $\mathrm{MB}(\mathrm{pH} 9)$ and $A G(\mathrm{pH} 3)$ on the degree of removal ([dye] $=5-10 \mathrm{mg} \mathrm{L}^{-1}, \mathrm{PANI}-\mathrm{HNTs}=10 \mathrm{mg}$ ).

diffusion models were calculated and are summarized in Table 3. A good correlation with the kinetic data explains the dye adsorption mechanism in the solid phase. The fitting with
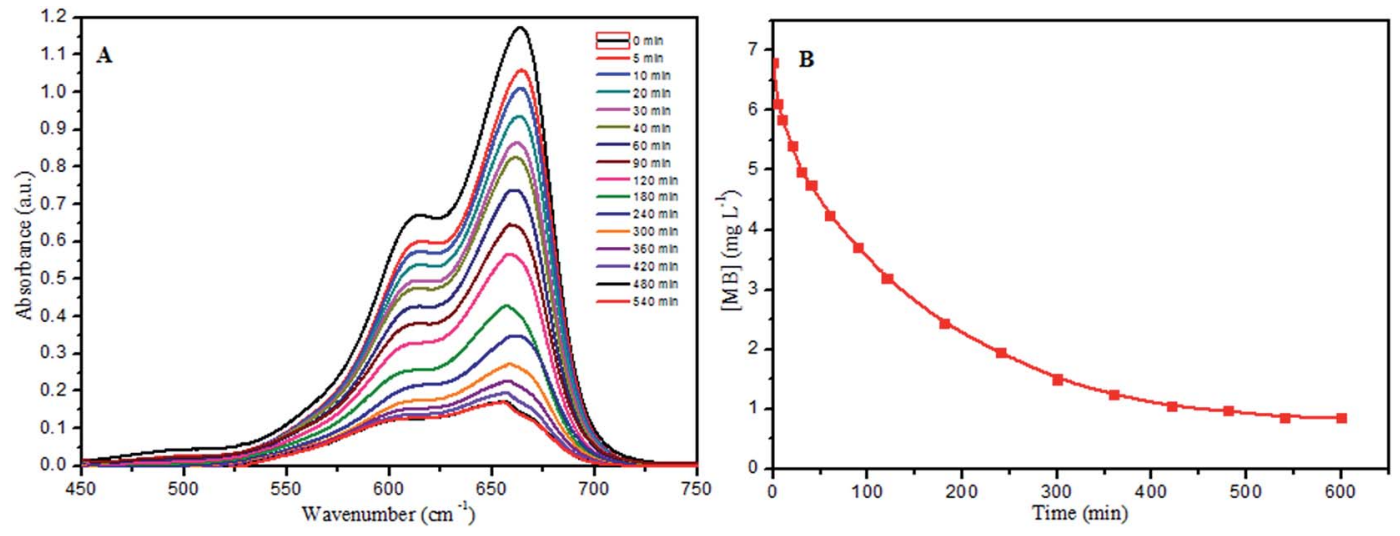

Fig. 6 (A) UV-vis spectra for MB adsorption from aqueous solution using PANI-HNTs, (B) the effect of contact time on the removal of MB dye (PANI-HNTs $=10 \mathrm{mg}$, $[\mathrm{MB}]=6.8 \mathrm{mg} \mathrm{L}^{-1}, \mathrm{pH}=9$ ). 
Table 3 Kinetic parameters for the adsorption of $M B$ and AG onto PANI-NTs [V: $0.01 \mathrm{~L}$, mass: $10 \mathrm{mg}$ ]

\begin{tabular}{llc}
\hline Kinetics models & AG at pH 3 & MB at $\mathrm{pH} 9$ \\
\hline Pseudo-first order & & \\
Experimental $q_{\mathrm{e}}\left(\mathrm{mg} \mathrm{g}^{-1}\right)$ & 36.67 & 59.4 \\
Theoretical $q_{\mathrm{e}}\left(\mathrm{mg} \mathrm{g}^{-1}\right)$ & 27.069 & 56.9 \\
$k_{\mathrm{s}}\left(\mathrm{min}^{-1}\right)$ & 0.009 & 0.008 \\
$R^{2}$ & 0.891 & 0.991 \\
& & \\
Pseudo-second order & & \\
Experimental $q_{\mathrm{e}}\left(\mathrm{mg} \mathrm{g}^{-1}\right)$ & 36.67 & 59.4 \\
Theoretical $q_{\mathrm{e}}\left(\mathrm{mg} \mathrm{g}^{-1}\right)$ & 38.744 & 40.74 \\
$k_{\mathrm{s}}\left(\mathrm{g} \mathrm{mg}^{-1} \mathrm{~min}^{-1}\right)$ & $8.9 \times 10^{-4}$ & 0.001 \\
$R^{2}$ & 0.994 & 0.996 \\
& & \\
Intraparticle diffusion $_{\text {Experimental } q_{\mathrm{e}}\left(\mathrm{mg} \mathrm{g}^{-1}\right)}$ & 36.67 & 59.4 \\
$C\left(\mathrm{mg} \mathrm{g}^{-1}\right)$ & 8.847 & 4.152 \\
$K_{\mathrm{i}}$ & 0.081 & 0.126 \\
$R^{2}$ & 0.853 & 0.967 \\
\hline
\end{tabular}

excellent linearity and a high correlation coefficient $\left(R^{2}=0.996\right.$ for $\mathrm{MB}$ at $\mathrm{pH} 9$ and $R^{2}=0.994$ for $\mathrm{AG}$ at $\mathrm{pH}$ 3) confirms the applicability of the pseudo-second order equation, as shown in Fig. 8. The pseudo-second-order kinetics for the adsorption of the cationic MB and the anionic AG dyes onto the PANI-HNTs substrate indicates that chemisorption (chemical adsorption) may be the rate-limiting step for the adsorption process. In chemisorption, the dyes stick to the PANI-HNTs adsorbent surface with the formation of a chemical bond.

\subsection{Equilibrium study}

Adsorption isotherms are mathematical models that describe the distribution of the adsorbate MB and AG dye species among the liquid and adsorbent, based on a few assumptions that are mainly related to the heterogeneity/homogeneity of the PANIHNTs adsorbent, the type of coverage and the possibility of interaction between the dye species. The most common sorption models used to fit experimental data are the Langmuir, ${ }^{6,38}$ Freundlich, ${ }^{1,4}$ and Temkin ${ }^{4,38}$ isotherm equations as follows.

The Langmuir isotherm:

$$
\frac{C_{\mathrm{e}}}{q_{\mathrm{e}}}=\frac{1}{q_{\mathrm{o}} k_{\mathrm{L}}}+\frac{C_{\mathrm{e}}}{q_{\mathrm{o}}}
$$

The Freundlich isotherm:

$$
\ln q_{\mathrm{e}}=\ln k_{\mathrm{f}}+\frac{1}{n} \ln C_{\mathrm{e}}
$$

The Temkin isotherm:

$$
q_{\mathrm{e}}=B \ln A+B \ln C_{\mathrm{e}}
$$

where $C_{\mathrm{e}}$ refers to the equilibrium concentration $\left(\mathrm{mg} \mathrm{L}^{-1}\right), q_{\mathrm{e}}$ refers to the amount of dye adsorbed at equilibrium $\left(\mathrm{mg} \mathrm{g}^{-1}\right)$ and $q_{\mathrm{o}}\left(\mathrm{mg} \mathrm{g}^{-1}\right)$ is the monolayer coverage capacity, $k_{\mathrm{L}}\left(\mathrm{L} \mathrm{mg}^{-1}\right)$ is the Langmuir isotherm constant, $k_{\mathrm{f}}$ and $n$ are the Freundlich constants, while $A$ and $B$ refer to the Temkin constants. The equilibrium data obtained were fitted to these three isotherm equations separately and the parameters were calculated and are shown in Table 4 along with the correlation coefficient for each fit (Fig. 9, S4 and S5†). The linear correlation coefficients show that the Langmuir isotherm best fits the data for MB and AG adsorption onto the PANI-HNTs.

According the obtained correlations $\left(R^{2}\right)$, the suspended powder system follows the Langmuir isotherm better than the Freundlich and Temkin models. Other reports involving PANI powders as adsorbents indicated the same tendency to obey the Langmuir model. ${ }^{8}$ The $q_{\max }$ for PANI-HNTs was found to be 69.4 and $57.8 \mathrm{mg} \mathrm{g}^{-1}$ for $\mathrm{MB}$ and $\mathrm{AG}$, respectively.

\subsection{Thermodynamic parameters}

Temperature is an important factor that strongly affects the adsorption of MB onto PANI-HNTs, ${ }^{46,47}$ and the adsorption capacity of MB dye decreased from 59.4 to 45.5, 39.9 and finally
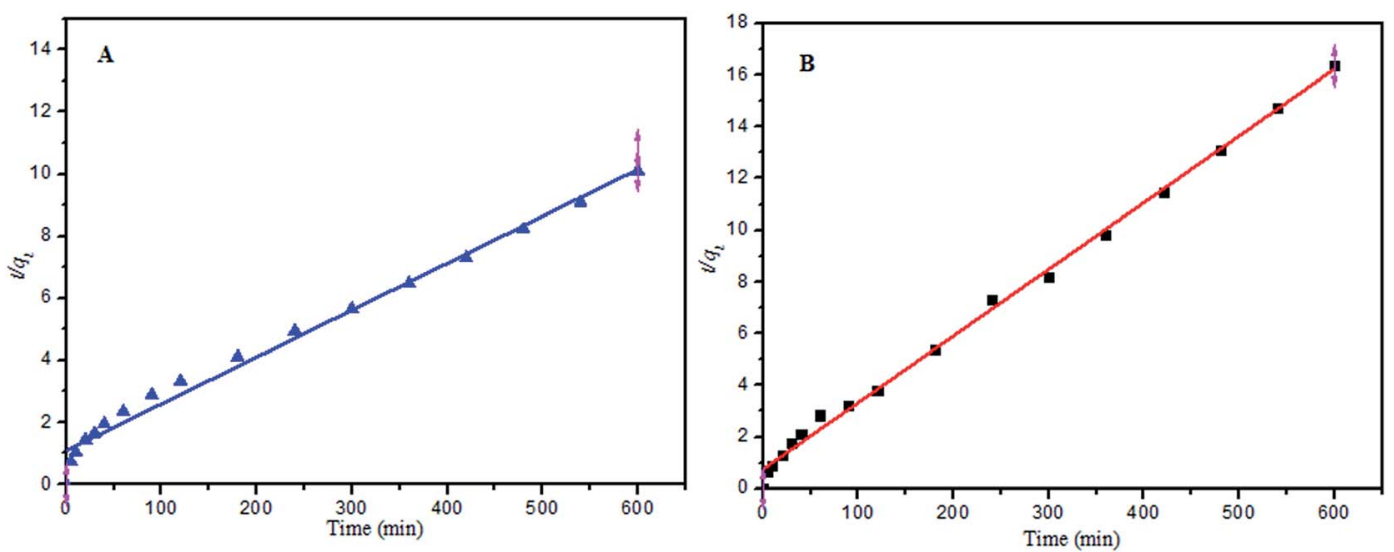

Fig. 8 (A) Pseudo-second-order reaction for MB adsorption onto PANI-HNTs at pH 9, (B) pseudo-second-order reaction for AG adsorption onto PANI-HNTs at $\mathrm{pH} 3$. 
Table 4 The Langmuir, Freundlich and Temkin isotherm parameters for the adsorption of MB and AG using PANI-HNTs [volume: $0.01 \mathrm{~L}$, time: $24 \mathrm{~h}$ and mass of PANI-HNTs: $10 \mathrm{mgl}$

\begin{tabular}{|c|c|c|c|c|c|c|c|c|c|}
\hline Dye & $q_{\mathrm{o}}$ & $k_{\mathrm{L}}$ & $R^{2}$ & $k_{\mathrm{f}}$ & $1 / n$ & $R^{2}$ & $B_{t}$ & $A_{t}$ & $R^{2}$ \\
\hline $\mathrm{AG}$ at $\mathrm{pH} 3$ & 57.87 & 17.99 & 0.987 & 48.569 & 0.10687 & 0.3594 & 414.4 & 3109.2 & 0.3613 \\
\hline
\end{tabular}
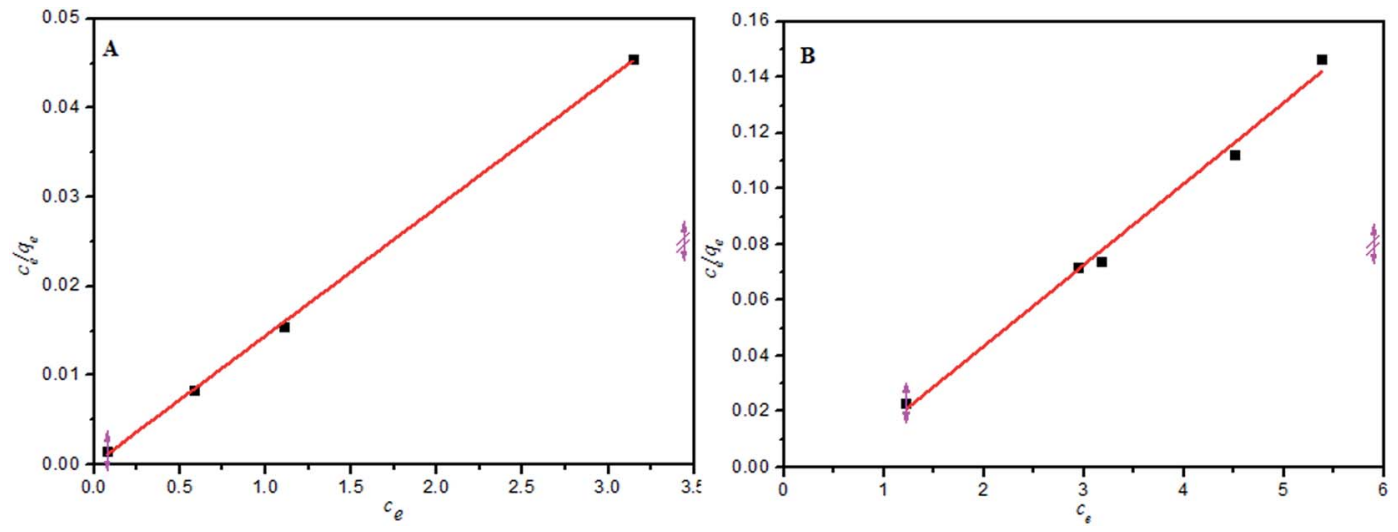

Fig. 9 (A) Langmuir isotherm for the adsorption of $M B$ (PANI-HNTs $=10 \mathrm{mg}$, $[M B]=5-10 \mathrm{mg} \mathrm{L}^{-1}$ ) at $\mathrm{pH}=9$, (B) Langmuir isotherm for the adsorption of $A G\left(P A N I-H N T s=10 \mathrm{mg}\right.$, $[A G]=5-10 \mathrm{mg} \mathrm{L}^{-1}$ ) at $\mathrm{pH}=3$.

to $43.7 \mathrm{mg} \mathrm{g}^{-1}$ due to a gradual temperature increase from 25 to 35,45 , and up to $55{ }^{\circ} \mathrm{C}$, respectively. This decreasing trend confirms that adsorption of MB dye on PANI-HNTs is an exothermic process. This may be due to weakening the residual forces between the active sites of the polymer and the dye, and between the adjacent dye molecules on the adsorbent as well. ${ }^{48}$ Furthermore, the equilibrium adsorption capacity, $q_{\mathrm{e}}$, of MB dye at different temperatures reached its maximum limit after ca. 400-500 min (as shown in Fig. 10).

From the previously stated data, some thermodynamic parameters can be calculated, such as the Gibbs free energy

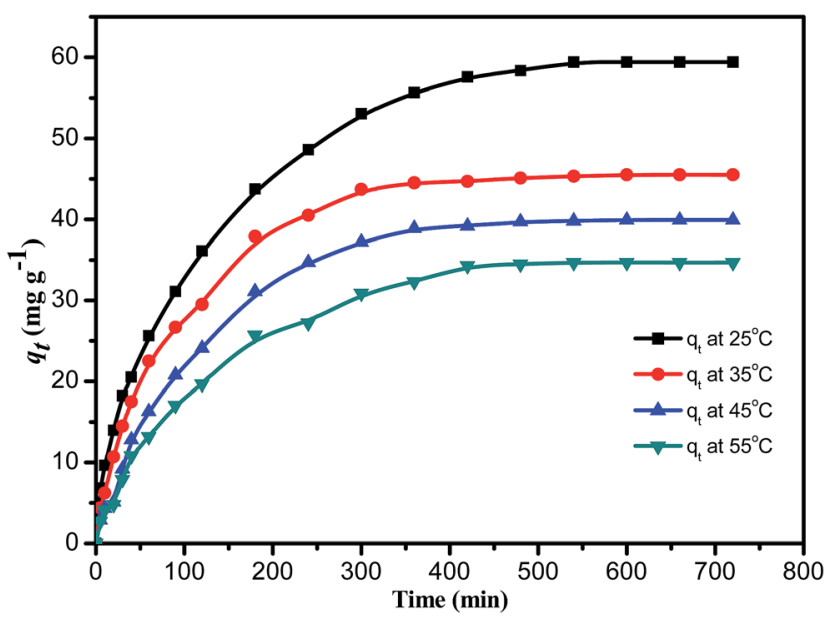

Fig. 10 The variation of adsorption capacity $q_{t}$ of $20 \mathrm{mg}$ PANI-HNTs toward $6.82 \mathrm{mg} \mathrm{L}^{-1} \mathrm{MB}$ solution at different temperatures. change $\left(\Delta G^{\circ}\right)$, enthalpy of adsorption $\left(\Delta H^{\circ}\right)$ and entropy change $\left(\Delta S^{\circ}\right)$, using the following equations and the values are collected in Table 5:

$$
\begin{gathered}
\Delta G^{\circ}=-R T \ln K_{\mathrm{d}} \\
\ln K_{\mathrm{d}}=-\frac{\Delta S^{\circ}}{R}-\frac{\Delta H^{\circ}}{R T}
\end{gathered}
$$

where $K_{\mathrm{d}}$ refers to the thermodynamic equilibrium constant, which is the ratio of the MB concentration on PANI-HNTs at equilibrium $q_{\mathrm{e}}$ to the remaining $\mathrm{MB}$ concentration in solution at equilibrium $C_{\mathrm{e}}{ }^{49,50}$

$$
K_{\mathrm{d}}=\frac{q_{\mathrm{e}}}{C_{\mathrm{e}}}
$$

The negative values of $\Delta G^{\circ}$ indicate that the adsorption of MB on PANI-HNTs is a feasible and spontaneous process under the experimental conditions used. ${ }^{51} \Delta H^{\circ}$ and $\Delta S^{\circ}$ can be determined from the slope and the intercept of the linear plot of $\ln K_{\mathrm{d}} v s .1 / T$ (Fig. 11, Table 5).

Table $5 \quad \Delta G^{\circ}$ values for MB dye adsorption at various temperatures

\begin{tabular}{lll}
\hline$T(\mathrm{~K})$ & $\ln K_{\mathrm{d}}$ & $\Delta G^{\circ}\left(\mathrm{kJ} \mathrm{mol}^{-1}\right)$ \\
\hline 298 & 4.2306 & -10.514 \\
308 & 3.0067 & -7.723 \\
318 & 2.853 & -7.566 \\
328 & 2.343 & -6.409
\end{tabular}




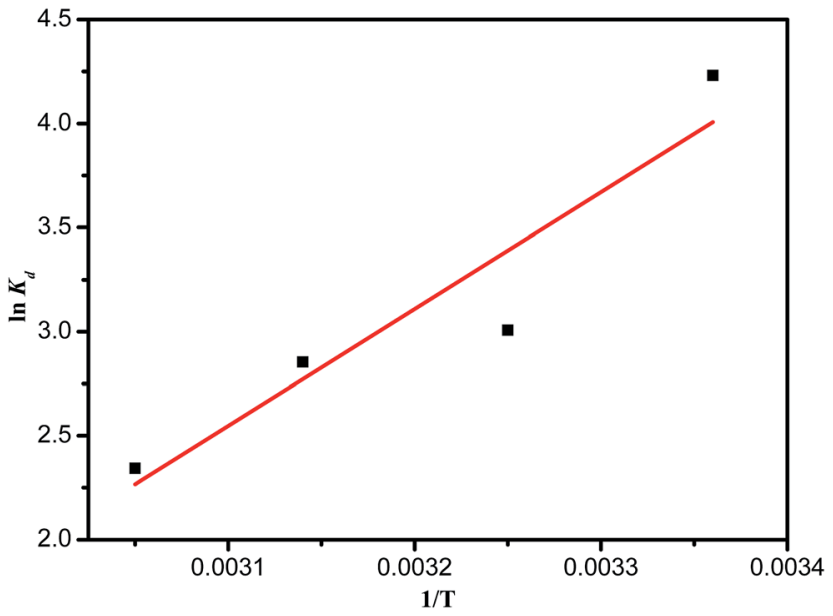

Fig. 11 Van't Hoff plot for MB adsorption on PANI-HNTs.

The results confirmed that the process of adsorption of $\mathrm{MB}$ is not exclusively physisorption. Generally, a process is considered as physisorption if the values of $\Delta H^{\circ}$ lie between 0 and $-20 \mathrm{~kJ} \mathrm{~mol}{ }^{-1} .^{52}$ The obtained negative $\Delta H^{\circ}$ $\left(-46.87 \mathrm{~kJ} \mathrm{~mol}^{-1}\right)$ confirms that the adsorption of $\mathrm{MB}$ dye on PANI-HNTs is an exothermic process, which matches the results of the study of the effect of temperature, in which the MB dye adsorption rate and capacity decreased with increasing temperature. The obtained negative $\Delta S^{\circ}(-124.05 \mathrm{~J}$ $\mathrm{mol}^{-1} \mathrm{~K}^{-1}$ ) indicates a decrease in the degree of disorder of the system at the solid-solution interface, and no significant change occurred in the internal structure of the synthesized PANI-HNTs during the adsorption process. This demonstrates the strong type of interaction between the MB dye and the PANI-HNTs adsorbent surface.

\subsection{Regeneration of the adsorbent}

The recovery of the synthesized PANI-HNTs, for use multiple times, was tested using $10 \mathrm{mg}$ of PANI-HNTs added to $6.8 \mathrm{mg} \mathrm{L}^{-1} \mathrm{MB}$ dye at $\mathrm{pH} 7$. In the first cycle, the adsorption of MB ended with an efficiency percentage of $81.7 \%$. After adsorption, the polymer sample was separated via centrifugation and the aqueous solution was drained slowly with a syringe. Regeneration of PANI-HNTs was accomplished by immersing the separated polymer in $100 \mathrm{~mL}$ of $1 \mathrm{~mol} \mathrm{~L}^{-1}$ ammonia for 30 minutes to activate all sites with negative charge in addition to the removal of the adsorbed MB dye. Afterwards, the polymer was separated via centrifugation then immersed in distilled water to remove the excess of ammonia.

For the first, second and third recycles, the adsorption of $\mathrm{MB}$ ended at 1.5 hours for all of them with efficiency percentages of $87.3 \%, 74.58 \%$ and $68.87 \%$, respectively (Fig. 12B). The first recycle has a higher efficiency $(87.3 \%)$ than the zeroth recycle (first cycle: $81.7 \%$ ) due to the activation of all the sites on the polymer treated with -ve charge, which led to strong electrostatic forces toward the adsorption of the cationic MB dye. In the second recycle, some of the polymers were lost due to incomplete separation, which led to decreasing adsorption efficiency of $\mathrm{MB}$ in addition to the partial saturation of the polymer with the adsorbed MB dye inside the nanotubes. The limited efficiency of the third recycle is assigned to more loss of polymer and more saturation of PANI-HNTs with the adsorbed MB dye.

\section{Conclusion}

In summary, we demonstrated the use of acid green crystals as a water-soluble soft-template for the fabrication of PANI-HNTs with a size range of 30-70 $\mathrm{nm}$ and a pore size of $7.4 \mathrm{~nm}$. After full characterization, the experimental adsorption studies showed that PANI-HNTs can be utilized for the adsorption of cationic and anionic dyes depending on the $\mathrm{pH}$ value. The adsorption of dyes onto PANI-HNTs followed a pseudo-second order kinetics model and the equilibrium data best fit the Langmuir model. The thermodynamic parameters indicate that the adsorption process is feasible, efficient, and spontaneous with a negative $\Delta H^{\circ}$ value. The PANI-NTs can be easily regenerated and reused for dye adsorption several times with good efficiency and high durability. The high adsorption capacity of PANI-HNTs toward both anionic and cationic dyes with a fast adsorption rate makes PANI-NTs a highly promising adsorbent for environmental sanitation.
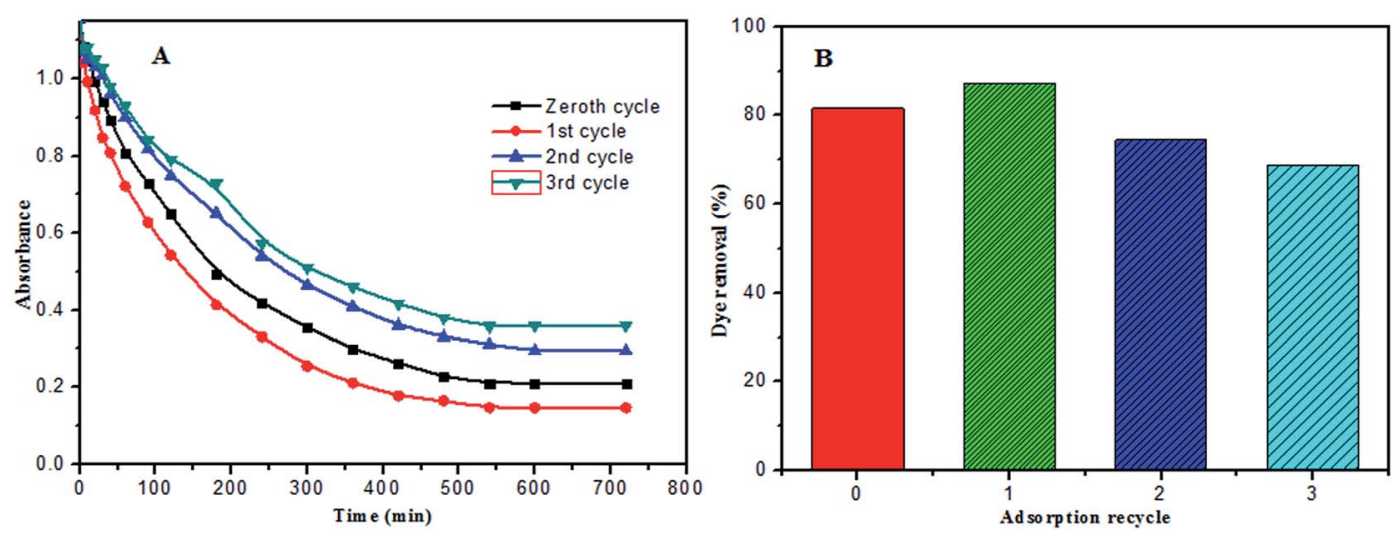

Fig. 12 Reusability of $10 \mathrm{mg}$ of PANI-NTs toward cationic dye removal. 


\section{Conflicts of interest}

There are no conflicts to declare.

\section{References}

1 O. A. Attallah, M. A. Al-Ghobashy, M. Nebsen and M. Y. Salem, RSC Adv., 2016, 6, 11461-11480.

2 G. Vijayakumar, R. Tamilarasan and M. Dharmendirakumar, J. Mater. Environ. Sci., 2012, 3, 157-170.

3 V. Sharma, P. Rekha and P. Mohanty, J. Mol. Liq., 2016, 222, 1091-1100.

4 J. R. Baseri, P. Palanisamy and P. Sivakumar, Eur. J. Chem., 2012, 9, 1266-1275.

5 B. N. Patra and D. Majhi, J. Phys. Chem. B, 2015, 119, 81548164.

6 M. R. Patil and V. S. Shrivastava, J. Mater. Environ. Sci., 2015, 6(1), 11-21.

7 M. M. Sk and C. Y. Yue, J. Mater. Chem. A, 2014, 2, 2830-2838. 8 M. M. Ayad and A. A. El-Nasr, J. Phys. Chem., 2010, 114, 14377-14383.

9 E. Ali, Nanostructured Conductive Polymers, John Wiley \& Sons, 2011.

10 A. Murat, K. Tolga and S. Sarac, Curr. Phys. Chem., 2012, 2, 224-240.

11 K. Bouhrira, F. Ouahiba, D. Zerouali, B. Hammouti, M. Zertoubi and N. Benchat, Eur. J. Chem., 2010, 7(1), 105110.

12 S. Edward and C. Jin-Woo, Nanomaterials, 2013, 3, 498-523. 13 B. Sambhu, K. Dipak, K. S. Nikhil and H. L. Joong, Prog. Polym. Sci., 2009, 34, 783-810.

14 A. N. Chowdhury, S. Jesmeen and M. Hossain, Polym. Adv. Technol., 2004, 15, 633-638.

15 D. Mahanta, G. Madras, S. Radhakrishnan and S. Patil, J. Phys. Chem. B, 2009, 113, 2293-2299.

16 D. Mahanta, G. Madras, S. Radhakrishnan and S. Patil, J. Phys. Chem. B, 2008, 112, 10153-10157.

17 M. M. Ayad and A. A. El-Nasr, J. Nanostruct. Chem., 2012, 3, 3. 18 M. M. Ayad, A. A. El-Nasr and J. Stejskal, J. Ind. Eng. Chem., 2012, 18, 1964-1969.

19 M. Ayad and S. Zaghlol, Chem. Eng. J., 2012, 204, 79-86.

20 H. Noby, A. El-Shazly, M. Elkady and M. Ohshima, J. Chem. Eng. Jpn., 2017, 50, 170-177.

21 H. Wang, L. Wang, R. Wang and X. Tian, J. Mater. Sci., 2011, 46, 1049-1052.

22 D. Chen, Y.-E. Miao and T. Liu, ACS Appl. Mater. Interfaces, 2013, 5, 1206-1212.

23 D. Sutar, S. Major, R. Srinivasa and J. Yakhmi, Thin Solid Films, 2011, 520, 351-355.

24 F. El-Dib, W. M. Sayed, S. Ahmed and M. Elkodary, J. Appl. Polym. Sci., 2012, 124, 3200-3207.
25 S. Chaudhari and P. Patil, Electrochim. Acta, 2011, 56, 30493059.

26 L. Ren, K. Li and X. Chen, Polym. Bull., 2009, 63, 15-21.

27 M. Wan, Adv. Mater., 2008, 20, 2926-2932.

28 A. D. Carswell, E. A. O'Rea and B. P. Grady, J. Am. Chem. Soc., 2003, 125, 14793-14800.

29 J. Huang and R. B. Kaner, J. Am. Chem. Soc., 2004, 126, 851855.

30 M. Li, W. Li, J. Liu and J. Yao, J. Mater. Sci.: Mater. Electron., 2013, 24, 906-910.

31 L. Pan, H. Qiu, C. Dou, Y. Li, L. Pu, J. Xu and Y. Shi, Int. J. Mol. Sci., 2010, 11, 2636-2657.

32 X. Haibing and T. Xutang, J. Mater. Chem., 2011, 21, 24632465.

33 T. Dai and Y. Lu, Macromol. Rapid Commun., 2007, 28, 629633.

34 M. Ayad, W. Amer and J. Stejskal, Thin Solid Films, 2009, 517, 5969-5973.

35 G. Ćirić-Marjanović, S. Mentus, I. Pašti, N. Gavrilov, J. Krstić, J. Travas-Sejdic, L. T. Strover, J. Kopecká, Z. Moravková and M. Trchová, J. Phys. Chem. C, 2014, 118, 14770-14784.

36 H. Xia and X. Tao, J. Mater. Chem., 2011, 21, 2463-2465.

37 H. T. S. Britton and R. A. Robinson, J. Chem. Soc., 1931, 14561462.

38 M. Ayad, N. Salahuddin, A. Fayed, B. P. Bastakoti, N. Suzuki and Y. Yamauchi, Phys. Chem. Chem. Phys., 2014, 16, 2181221819.

39 B. I. Nandapure, S. B. Kondawar, M. Y. Salunkhe and A. I. Nandapure, Adv. Mater. Lett., 2013, 4, 134-140.

40 M. Trchová and J. Stejskal, Pure Appl. Chem., 2011, 83, 18031817.

41 H. Ding, J. Shen, M. Wan and Z. Chen, Macromol. Chem. Phys., 2008, 209, 864-871.

42 Y. Li, X. Zhao, Q. Xu, Q. Zhang and D. Chen, Langmuir, 2011, 27, 6458-6463.

43 Y.-S. Ho, J. Hazard. Mater., 2006, 136, 681-689.

44 Y.-S. Ho and G. McKay, Process Biochem., 1999, 34, 451-465. 45 I. Uzun, Dyes Pigm., 2006, 70, 76-83.

46 L. Abramian and H. El-Rassy, Chem. Eng. J., 2009, 150, 403410.

47 A. Ebrahimian and E. Saberikhah, Cellul. Chem. Technol., 2013, 47, 657-666.

48 A. E. Ofomaja and Y.-S. Ho, Dyes Pigm., 2007, 74, 60-66.

49 M. Y. Nassar, T. Y. Mohamed, I. S. Ahmed and I. Samir, J. Mol. Liq., 2017, 225, 730-740.

50 M. Y. Nassar, E. I. Ali and E. S. Zakaria, $R S C A d v ., 2017,7$, 8034-8050.

51 M. Ertaş, B. Acemioğlu, M. H. Alma and M. Usta, J. Hazard. Mater., 2010, 183, 421-427.

52 Y. Zhang, F. Yu, W. Cheng, J. Wang and J. Ma, J. Chem., 2017, 2017. 